\title{
Fútbol: ¿catalizador de inseguridad? Análisis del fenómeno social y su incidencia en la seguridad
}

\author{
Andrés Felipe Briceño Rodríguez* \\ (andres.briceno@correo.usa.edu.co)
}

Artículo corto recibido el 04/11/2014 y aprobado el 19/12/2014

Cómo citar este artículo:

BRICEÑO RODRÍGUEZ, Andrés Felipe (2014). "Fútbol: ¿catalizador de inseguridad? Análisis del fenómeno social y su incidencia en la seguridad”. En: Transpasando Fronteras, núm. 6, pp. 141-156. Cali, Colombia: Centro de Estudios Interdisciplinarios, Jurídicos, Sociales y Humanistas (CIES), Facultad de Derecho y Ciencias sociales, Universidad Icesi.

\section{Resumen}

Desde sus orígenes, el balón pie se ha visto relacionado con la seguridad en varias de sus dimensiones, de manera intrínseca, reciproca e incluso complementaria. Sin embargo, por la popularidad de la cual goza, y su capacidad para conglomerar efervescentes aficionados, se ha malinterpretado su rol dentro de las sociedades donde éste es aceptado. Cabe preguntarse si este catalizador de grandes pasiones resulta entonces una vulneración de dicha seguridad en el desenvolvimiento de sus interacciones. ¿Es el fútbol un generador de violencia? ¿La seguridad se ve afectada por este deporte? La relación de

* Estudiante de Política y Relaciones Internacionales Universidad Sergio Arboleda (Colombia). Representante Estudiantil Escuela de Política y Relaciones Internacionales. Web Máster y Community Manager Red Intercol (Red colombiana de Relaciones Internacionales), Communiy Manager en Indignados Colombia Oficial. Columnista Revista Sócrates. Fundador, columnista y editor de Punto Crítico; una visión controversial del Fútbol y la Política, Columnista Pase al Vacío. Locutor en los programas "La Banda” y "Hora LATAM", para USARTV (Radio Virtual Sergio Arboleda). 
estos dos elementos resulta innegable, evidenciando vulnerabilidades y sensibilidades, por lo cual, este documento pretende analizar su correspondencia, y las externalidades de la misma. Para ello, se realizará un análisis breve de las principales prevaricaciones de la seguridad, y contrastarla con una mirada desde las relaciones generadas a partir de jugadores profesionales que migraron a las ligas más importantes del planeta, producto de la exportación de los mismos al fútbol europeo (puntualmente, Alemania, España, Italia y Portugal). Las cuales como saldo no sólo abren mercados e incentivan la presencia nacional en el viejo continente, sino tienen una serie de repercusiones encarnadas en la labor dentro y fuera de las canchas de cada uno de los jugadores, visibilizando a Colombia dentro de una agenda Soft Power, y retribuyendo beneficios económicos y sociales a sus regiones de origen (escuelas de fútbol, fundaciones, presencia mediática y gubernamental).

\section{Palabras clave:}

Seguridad económica, seguridad social, seguridad alimentaria, fútbol, deportivización.

\section{Introducción}

Los Hooligans como perfecto ejemplo de violencia e inseguridad, se entienden históricamente como el yacimiento de lo que sería la violencia colectiva en el fútbol ya reglamentado e institucionalizado, y también, de los Barristas, su adaptación latinoamericana. Asimismo, son recordados desde el fútbol como los responsables de varios actos vandálicos como las tragedias en los estadios de Heysel (Bélgica) en 1985, que dejó treinta y nueve muertos, y de Hillsborough (Inglaterra) en 1989, con noventa y seis víctimas mortales. Esta serie de sucesos hicieron que el fútbol inglés tocara fondo y constituyeron elementos de relación directa con la seguridad y la vulneración de la misma. Sin embargo, su nacimiento viene propiamente de una crisis económica con un matiz ideológico, que curiosamente evoluciona con implicaciones raciales y nacionalistas entre otras (Briceño, 2008).

Así pues, se materializó el vandalismo del fútbol conocido por hooliganism, entendido también como la "enfermedad británica”. El auge de la violencia ligada al deporte actual no se limita ni a la conducta de los aficionados al fútbol ni a Inglaterra y su fenómeno ya casi extinto. Posiblemente el ensanchamiento de la violencia y la dilatación del fenómeno se incumba a una "tendencia autodestructiva dentro del deporte en todo el mundo, principalmente como resultado de las peticiones de búsqueda de sensaciones por parte de los espectadores" (Cayuela Maldonado, 1997:4). 
Tras la crisis económica de Inglaterra, el desempleo que agobiaba a las clases trabajadoras, y, como alternativa a los frustrados grupos sindicales, surgen estos grupos como representación de una clase social dedicada a la mano de obra básica como actividad económica principal, aunque sin tanta trascendencia (Briceño, 2008).

Estos mismos grupos encontraron en el fútbol, un refugio de la realidad nacional, generando éste fatal matrimonio. Del mismo modo, resultaba común relacionar hooligans con grupos neonazis, dado la influencia de estos últimos sobre su identidad los hooligans ocasionaron ataques a personas de color, como ocurrió en Marsella donde tunecinos y franceses se enfrentaron a los ingleses dejando todo destruido a su paso (Recaséns, 2008:56). Así pues, "los grupos violentos hacen de la violencia y el altercado su razón de ser y el elemento aglutinador del colectivo" (UNISPORT, 1993).

Una de las grandes conclusiones tras la investigación previa a las medidas tomadas por Margaret Thatcher para controlar el hooliganism resultó ser el reconocimiento del problema como un fenómeno sociocultural del país y no propio del fútbol, lo cual de entrada ya permite aclarar la relación entre la violencia y el deporte con nuevas posibles variables (Briceño, 2013).

Hoy en día, la Liga inglesa es una de las más cotizadas, y su desarrollo se da libre de violencia, en grandes estadios sin cercas, con un público sentado en sillas numeradas donde los gritos y aplausos son las únicas expresiones de euforia lo cual en principio desligaría al balón pie de la violencia (Recaséns, 2008:56).

Sin embargo, no hay que desconocer que los deportes en general, son uno de los fenómenos más populares de nuestro tiempo. Son en éstos, dónde se producen y expresan algunos de los grandes valores de la sociedad contemporánea. Miguel de Moragas afirma que "las modernas historias sobre la bondad y la maldad, el éxito y el fracaso, la suerte y la desgracia, la victoria y la derrota, lo propio y lo ajeno, la identidad colectiva (...) encuentran en la narración deportiva sus expresiones más populares" (Cayuela Maldonado, 1997:3). Por ende, resulta un adaptable y eficiente canal de expresiones sociales, más aún el fútbol, al ser el deporte gozoso de mayor popularidad. No en vano Xavier Medina (1994) afirma que "el fenómeno deportivo está estrechamente vinculado a la realidad social y cultural, hasta el punto que se transforma con ella". 
El deporte resulta ser entonces, un instrumento de transmisión o canalización si se quiere, de cultura y de valores básicos del propio marco cultural en el que se despliega. Como producto social, la práctica deportiva se convierte en un elemento clave de socialización. Medina entiende por tal concepto:

\begin{abstract}
"la interiorización por parte de los individuos de aquellos elementos que integran un sistema sociocultural determinado y que permite las interrelaciones que hacen posible dentro del grupo la generación de vida compartida. Elementos que desde esta perspectiva, producen y recrean la diversidad grupal" (Cayuela Maldonado, 1997:10).
\end{abstract}

La violencia en el deporte definida de manera amplia, puede entenderse como el "infligir voluntariamente daños físicos o de otro orden a personas u objetos relacionados directamente con encuentros deportivos" (Parlamento Europeo, 1987). Actos que pueden ser considerados detalladamente como una serie de prácticas que implican violencia, física, psicológica y moral. Los juegos olímpicos de Berlín de 1936 sirven de ejemplo de ello, pues se encontraron bajo el auge glorificador del nazismo, compuesto por su ideología totalitaria y racista. Considerándose así como una manifestación de violencia, de modo similar al asesinato de atletas israelíes durante los Juegos de Múnich de 1972 (Cayuela Maldonado, 1997:4).

Sumado a lo anterior, la conducta del ser humano de por sí hace un llamado a la controversia, y en una suerte de humanidad, requiere del conflicto (Di Giano, 2005:33-38). Desde la concepción individual a partir de la construcción capitalista de las libertades se da sustento a la continua competencia, conducta parida de la evolución de la supervivencia. Incluso, y desde el individualismo (Cayuela Maldonado, 1997), la naturaleza humana cataliza la necesidad de un enemigo, como lo afirmaría Karl Schmidt. Por tanto, la proclividad a la violencia a partir del deporte donde no sólo se potencian las emociones sino se simulan las disputas mediante la competencia, resulta el escenario perfecto para materializar las contiendas humanas (Durán González, 2012).

Siguiendo esta línea, Josep Gomis afirma que la realidad demuestra que la violencia no es un fenómeno aislado, sino que ha tenido una evolución muy parecida en todos los países de nuestro entorno cultural. No obstante, se señala a la vez que "la violencia 
relacionada con el deporte ha superado ampliamente el estricto marco de los recintos de fútbol para salir a la calle, afectando a barrios enteros" (Gomis, 1989). Por tanto, la relación del fútbol y la seguridad se va aclarando, destacando que propiamente lo que hace el deporte rey es canalizar, más no generar vulneraciones a la seguridad. Por otra parte, la seguridad como concepto posee otras connotaciones, con las cuales el fútbol también posee otro tipo de relación, tal vez no causal, pero sí delimitadora.

\section{Las dimensiones de la seguridad con el deporte}

Para una mayor comprensión de lo que implica lo expuesto anteriormente, resulta necesario abordar de manera conceptual los componentes que harán las veces de guía para explicar el fenómeno y su relación con las diferentes esferas de la concepción de seguridad y su vulneración.

En este orden de ideas, la seguridad como concepto comprende una amplitud de definiciones que han llevado partes a subdividirla en diferentes categorías. Para comprender como se ve relacionado con el fútbol como fenómeno social con la seguridad, será necesario realizar un bosquejo conceptual a manera de preámbulo, que permita esclarecer su coyuntura. Pese a sus detractores, para el presente documento nos centraremos en tres segmentaciones básicas para asimilar la temática: La seguridad Social, la Seguridad Económica y la Seguridad Alimentaria.

En la diacronía de la humanidad la seguridad ha constituido una constante preocupación que de manera evolutiva ha ido acaparando varios campos, que han llevado a las búsqueda de diferentes formulas para combatir la miseria, la ignorancia, las enfermedades, el hambre, y en general, la protección de la vida del hombre, independientemente de su religión, raza, credo o nacionalidad (Alvarez Soberanis:1-2).

Por su parte, la seguridad social, se refiere concretamente a la protección que una sociedad proporciona a los individuos y los hogares para asegurar el acceso a la asistencia médica y garantizar la seguridad del ingreso, en particular en caso de vejez, desempleo, enfermedad, invalidez, accidentes del trabajo, maternidad o pérdida del sostén de familia, la cual a su vez se constituye como un derecho fundamental acorde a los Convenios de la OIT y en los instrumentos de la ONU (Organización Internacional del Trabajo, 2001). 
De la cual se desprende la seguridad económica, la cual hace referencia a la capacidad de disponer y usar de forma independiente una cierta cantidad de recursos económicos regulares y en montos suficientes para asegurar una buena calidad de vida (Guzmán, 2002). También puede entenderse como la capacidad de las personas de disponer de bienes en general, económicos y no económicos, para generar agregación a la calidad de vida y disponer de independencia y dignidad en temas como el envejecimiento (Huenchuan y Guzmán, 2006). Por consiguiente complementa la seguridad alimentaria, entendida como la disponibilidad física de los alimentos, su acceso físico y económico además de su apropiada utilización (FAO, 2011).

Aclarado lo anterior, resulta necesario tener en cuenta para más adelante relacionar el concepto de Deportivización, acuñado por Norbert Elias y Eric Dunning para hacer referencia al aporte a las condiciones de vida del ser humano a través de prácticas deportivas comprendiendo las dinámicas industriales de la sociedad moderna y el capitalismo.

Elias y Dunning mencionan que la deportivización de la sociedad forma parte del proceso complejo y contradictorio de consolidación del capitalismo, en tanto organización hegemónica productiva del mundo y en tanto organización social, que junto con el proceso de industrialización crearon diferentes formas y condiciones de vida en las que las personas y los grupos sociales han establecido y establecen diferentes tipos de relaciones y desempeñan distintos tipos de trabajo (Huerta Rojas, 2002:48).

Que en últimas, y retomando la idea de la necesidad del conflicto y las disputas del ser humano, el concepto de la deportivización también aporta a la contención y desahogo de tal necesidad humana. Pues al simular de manera competitiva, recreativa y ociosa las disputas de manera reglamentada, se genera una descarga de tal deseo de conflicto evitando que en un campo no deportivo o futbolístico se lleven a cabo tales contiendes (Cayuela Maldonado, 1997).

\section{Relacionamiento historicista}

Las repercusiones del deporte comprendiendo lo ya expuesto, han llevado a agenda el tema a nivel gubernamental. A partir de 1968 en el marco fundamental de las constituciones modernas, el Estado-Nación concibe y aplica la importancia social de manera prematura, haciendo del deporte una herramienta en su accionar que favorece a la atención de 
necesidades y al mejoramiento de la calidad de vida de los individuos. Con la intención de profundizar así, en una suerte de funcionalidad política abarcada desde las políticas públicas de manera posterior, con un creciente número de objetivos no deportivos como producto tácito. A consecuencia de lo anterior, las políticas públicas del deporte resultan ser la válvula de escape de conflictos sociales, haciendo de salvaguarda del orden público, y adicionalmente la higiene poblacional de una comunidad con las externalidades positivas que esto conlleva (Bloyce y Smith, 2012:1).

Así las cosas, el fútbol constituye a su vez el deporte Rey, el más popular y/o de mayor afluencia, transmisión, audiencia y número de seguidores y aficionados. Por consiguiente, cada vez tendrán más importancia los estudios sobre el fútbol como fenómeno social al resultar el mecanismo más operante que comprende la participación de las masas de manera fervorosa si se tiene en cuenta el margen de acción de manera comparada con otros deportes (Durán González, 2012:13).

Como diría Diego Armando Maradona, "La pelota no se mancha". El llamado es a prestar mayor atención a la esférica, más no a satanizarla. En sí, dada su capacidad masificadora, el fútbol resulta pues, tal canal de desahogo de las furias abstractas y concretas del ser humano moderno, y de ahí su potencial peligrosidad, la cual en últimas no ha logrado aún alcanzar "las cotas de violencia histórica provocada por los integrismos religiosos, políticos, sociales o culturales” (Durán González, 2012:13).

Por su parte, el director del Instituto de Relaciones Internacionales y Estratégicas Pascal Boniface (IRIS) de París, afirma que el balompié resulta, ya que facilita el enfrentamiento simbólicamente limitado necesario ante la inminente naturaleza de confrontación del ser humano (Boniface, 2006). Evitando así riesgos políticos, canalizando la "eliminación" del rival de manera abstracta y temporal.

El fútbol no solo puede entenderse como catalizador de enfrentamientos, porque los jugadores pueden ser instrumentos de baja política y verdaderos expositores del soft power. Por ejemplo, después de clasificarse Costa de Marfil para el Mundial de Alemania 2006, en una sentida rueda de prensa, Didier Drogba se dirigió a todos sus compatriotas junto a sus compañeros de selección y pidió a las facciones del norte y del sur enfrentadas que dejaran las armas y pusieran fin al conflicto que ha arrasado su país, llevando al fin del conflicto (Minuto 116, 2014): 
"Ciudadanos del norte, del sur, del este y el oeste: pedimos de rodillas que se perdonen los unos a los otros. Un gran país como el nuestro no puede rendirse al caos. Abandonen las armas y organicen unas elecciones libres" (Didier Drogba).

\section{Estudio de Caso Local (Buenaventura)}

No en vano, Drogba fue nombrado como 'Embajador de Buena Voluntad' por parte de las Naciones Unidas (Minuto 116, 2014).El simbólico reconocimiento concibe cierta evocación que más allá de su denominación, permite entrever ciertas funciones implícitas que no se limitan a lo deportivo, pero que por sus salvedades requieren iniciativas individuales ante la ausencia de voluntad política.

Como veremos a continuación, en el caso colombiano hay tímidos intentos de hacer uso del fútbol para abordar y enfrentar problemas en las distintas dimensiones de seguridad analizadas con anterioridad.

Por consecuencia, y a manera de casualidad, muchos de los deportistas colombianos y sobretodo futbolistas, han surgido de zonas deprimidas y regiones muy pobres. Buenaventura es uno de esos nichos, ubicada en el suroccidente de Colombia, es el municipio más grande del departamento de Valle del Cauca, sus $6.078 \mathrm{~km} 2$ de superficie representan la tercera parte del área departamental, y el segundo con más población, 324.207 habitantes, según el censo realizado por DANE, en 2005.

Fundada el 14 de julio de 1540, por Juan de Ladrilleros, teniente del adelantado Pascual de Andagoya, venido a América con Vasco Núñez de Balboa en 1515, en cumplimiento de una orden de éste. Las tierras habitadas por los indios buscajaes y noamaes en la isla de Cascajal situada en la bahía de Buenaventura, una de las más seguras para la llegada y salida de barcos de gran calado sobre el Pacífico resultó ser el perfecto cómplice estructural para el arribo de los colonizadores. Como su fundación se dio el día de San Juan de San Buenaventura y su conmemoración, esta región recibe tal nombre. La tranquilidad de sus aguas y su bahía es reconocida desde la década de los 50 como el primer puerto marítimo de Colombia (Suárez Reyes, 2010:2494).

La brecha existente entre las condiciones socioeconómicas de los habitantes de la periferia y los del interior en Colombia resulta notoria y propia de países latinoamericanos gozosos de contrastes y brechas sociales, tal y como ocurre con la Costa del Pacífico colombiano. Buenaventura no resulta ajeno a esta realidad, pues a pesar de 
contar con el puerto más importante del país, Buenaventura es el municipio de mayor pobreza del Valle del Cauca. No basta con el auge en la movilización de carga que ha traído el creciente mercado mundial y la tentativa de expansión portuaria, infraestructural. Pues los altos índices de pobreza y los yacimientos de violencia y grupos armados al margen de la ley, resultan en un estancamiento regional que ante el abandono estatal, las condiciones de salubridad y el denotan la miseria de ésta región, y por ende, un ambiente propicio para la generación de un circulo vicioso con la inseguridad como protagonista (Suárez Reyes, 2010:2488-2489).

Pues bien, ésta región multicultural, de grandes riquezas naturales, minerales, actividad portuaria y pobreza extrema, también es exportadora de futbolistas que han forjado la historia del fútbol colombiano. Jugadores como Freddy Rincón, Adolfo "el Tren" Valencia y Marino Klinger - entre otros- han sido paridos en el municipio del Valle del Cauca, y encontraron en el deporte rey la oportunidad de auto-realización.

Jugando descalzos en áridas canchas de tierra y escombros como escenario deportivo, además de humildes escuelas de fútbol como el Deportivo Buenaventura, surgieron profesionales como ocurrió con los hermanos Rincón, iniciando un proceso de retroalimentación exponencial en las diferentes dimensiones de seguridad planteadas, generando rompimiento progresivo de cinturones de miseria de manera intrínseca y disipada, iniciando una era con posible metástasis que salta de generación en generación (Duque Soto, 2011).

Y se hace referencia a una retroalimentación exponencial comprendiendo el corto, mediano y largo plazo a partir de lo ya anteriormente expuesto. En el corto plazo, la ocupación inmediata de los jóvenes ociosos ya repercute en la seguridad de una comunidad siendo que la juventud inoficiosa y carente de oportunidades es proclive a delinquir y a caer en diferentes vicios. En el mediano plazo, el aporte que genera la práctica constante de un deporte se remite al bienestar y la calidad de vida, además de aliviar la higiene poblacional concretando así, la deportivización como enfoque de seguridad. Se habla de un largo plazo, y del carácter exponencial, comprendiendo las diferentes dimensiones de seguridad a partir de la ejemplificación del "coloso de Buenaventura", quien como muchos de los jóvenes que entrenan deshidratados a diario "buscan el sueño de ser jugador profesional para rescatar a sus familias de la pobreza”, como afirmaría 
Cristóbal Rodríguez, entrenador actual del Buenaventura. Éste incentivo llevó a que Freddy no sólo debutara en el fútbol profesional colombiano, sino hizo que abriera mercados para sus compatriotas en los más importantes escenarios mundiales, como lo son Brasil, España y Alemania (Duque Soto, 2011).

Paralelamente, Rincón quiso retribuir de cierta manera su sobresaliente superación personal, iniciando un proyecto de la mano del Médico Ochoa único sobreviviente de la época del Dorado: "Los colositos de Buenaventura". Ésta escuela de fútbol al igual que varias iniciativas individuales, han generando un nicho de nuevas oportunidades para repetir la historia de Freddy Eusebio.Sin embargo el caso de Rincón no es el único, ni el más visible. Presentemente, existen varias escuelas, como la escuela Sporting Club con cancha de barro, o la Fluminense del barrio Bolívar, que sin pavimentación ni vías de acceso recibe más de 100 niños semanalmente para entrenamientos. Actualmente, los ex profesionales Osman López, Bonner Mosquera, Roger Moreno y Juan Reyes dirigen La Fundación "Vení jugá”, la cual destina aportes a 11 escuelas de fútbol bonaverenses y seleccionan talentos, involucrando casi a mil niños en el proceso, aumentando el margen de acción que no sólo promueve la seguridad en todas sus dimensiones, sino atiza potenciales "colosos" que de seguro aportaran al ciclo ya planteado (retroalimentación exponencial) una vez profesionales y consagrados como figuras del deporte nacional y/o mundial haciendo de este fenómeno de deportivización algo creciente (Duque Soto, 2011).

Así pues, el fútbol genera un marco de oportunidades que en ausencia estatal, no genera ni la educación ni las oportunidades laborales, evitando la criminalidad y aportando de manera individual a las diferentes dimensiones de seguridad de las familias bonaverenses. En la actualidad, jugadores de Buenaventura como Luis Fernando Mosquera y Lionard Vásquez (Millonarios), William Arboleda del Independiente Medellín, o Félix Antonio García la Equidad Club Deportivo, actúan en el fútbol profesional colombiano, generando remesas de manera prematura con salarios que superan los de un individuo promedio con cualquier pregrado. Sumándose a la lista de los frutos deportivos de Buenaventura, como lo fueron los ya nombrados, Delio 'Maravilla' Gamboa, Víctor y Teófilo Campaz, Nicolás Lobatón, Adelmo 'Achito' Vivas, Osman López, sin contar con los que siguen en el anonimato y/o categorías inferiores (Duque Soto, 2011). 


\section{En la actualidad}

Freddy Rincón hizo parte de esa primera generación que abrió mercados para sus compatriotas de manera histórica, en países como Italia, Portugal, España y Alemania. Así pues, hoy en día el número de colombianos en el exterior crece exponencialmente, con presencia en mayor número de ligas (incluso Asia o naciones árabes), en todas las categorías y en los más importantes clubes del planeta como principales protagonistas.

Varias de estas figuras, también tienen un origen humilde, como Carlos Bacca actual campeón de la UEFA, quien fue vendedor de pescado y ayudante en los buses. Y al igual que $E l$ Coloso han querido hacer una retribución a su comunidad, o al fútbol como tal.

Para nombrar sólo algunos ejemplos, es necesario hacer referencia a figuras como Juan Guillermo Cuadrado, figura de la Fiorentina de Italia y originario de Necoclí, humilde municipio de la subregión del deprimido Úraba. Ni que decir de Jackson Martinez, goleador del Porto de Portugal, y nacido en el Chocó, que como es bien sabido, es una de las regiones de mayor abandono estatal y pobreza arraigada.

Por su parte, Juan Guillermo quien obtuvo el mayor número de asistencias en el pasado mundial de Brasil 2014, es fundador de la Fundación Fútbol para el futuro, abordando por medio del fútbol necesidades que van desde la salud y la alimentación, hasta el bilingüismo para jóvenes de bajos recursos (Fútbol para el futuro, 2012).

Su origen y función social no solo soporta la idea de la deportivización cíclica y exponencial, sino tiene definidos concretamente ciertos objetivos como se plantea claramente en su plataforma web:

Fútbol para el Futuro nace como proyecto de desarrollo social en marzo de 2013 y constituido como organización sin fines de lucro en octubre del mimo año en aras de lograr 2 objetivos fundamentales:

- Llevar a cabo programas de fútbol social cuyo objetivo es ayudar familias que tengan dentro de su núcleo familiar niños y niñas, utilizando el deporte y la educación como vehículos de crecimiento y desarrollo personal.

- Liderar programas de mejoramiento del fútbol Colombiano como deporte de mayor influencia en nuestra sociedad para lograr que todos aquellos actores como lo son jugadores amateurs, jugadores de elite, ex profesionales, directo- 
res técnicos, directivos, padres de familia, árbitros, hinchas, instituciones de educación y por supuesto el estado, cuenten con un aliado para mejorar la variables humanas y técnicas de las próximas generaciones asociadas al mundo del Fútbol (Fútbol para el futuro, 2012).

Además, aporta a la seguridad social y económica planteándose como propósito la medición de salida, la graduación de sus integrantes en los ciclos de formación académica (primaria, secundaria y media vocacional) además de la habilitación para la empleabilidad y las habilidades ciudadanas, contando con varios patrocinios públicos y privados (Fútbol para el futuro, 2012).

Generando un aporte a la sociedad similar al de Jackson Martinez, quien a partir de su experiencia en las fundaciones y escuelas como Los Virtuosos y la Escuela de Formación Los Papeticos, fundó su propia fundación luego de que se superó e incluso llegó a hacer parte de la Selección Chocó del Pony Fútbol con un gran desempeño futbolístico desde estas dinámicas formativas del deporte (Fundación Deportiva Jackson Martinez, 2014).

De esta manera, la fundación de Jackson ataca las falencias de la seguridad de manera directa y sistemática en zonas deprimidas, demostrando una vez más que el fútbol en su relación con la seguridad no se da como catalizador sino más bien a manera de complemento. Más concretamente podría inferirse tal conclusión a partir de sus logros y objetivos como se vislumbra en su portal:

Somos una organización sin ánimo de lucro que nace el 9 de noviembre del 2012 por la iniciativa del jugador profesional de talla internacional Jackson Martínez, con el objetivo de contribuir a que los niños y jóvenes, menos favorecidos de la sociedad, tengan una posibilidad de crecer en el deporte a la par de su estudio.

Alcanzar este reto, nos enfocamos en diferentes regiones del país a través de un modelo de trabajo "Apoyando el talento", que busca que la práctica deportiva se realice sin restricciones de ninguna indole.

Enmarcados en esta iniciativa innovadora hacemos del deporte y la educación una herramienta fundamental para la transformación social de Colombia (Fundación Deportiva Jackson Martinez, 2014). 
La estrella colombiana, quien marcó dos tantos en el mundial y encabeza la lista de goleadores de Portugal, cuenta con el apoyo de de diferentes jugadores profesionales además de la empresa privada. A su vez, de manera constante realiza actividades como subastas de artículos de jugadores famosos y solicita el material de entrenamiento e indumentaria de sus diferentes rivales y compañeros para dotar a sus acogidos.

A estas iniciativas se le suman muchas otras como Colombia te quiere ver de Iván Ramiro Córdoba, ídolo del Inter de Milán, Italia, o las Escuelas de Carlos el "Pibe" Valderrama por sólo mencionar algunas.

Por tanto, no resulta ingenuo pensar en que los niños beneficiados por todo este tipo de programas a futuro estarán realizando una actividad similar, más teniendo en cuenta el momento histórico que atraviesa el deporte nacional, además de la valorización y prestigio de la cual gozan los deportistas colombianos. En lo que respecta a tales figuras y estrellas deportivas, no sólo están haciendo las veces de embajadores fuera del país, también están generando un accionar social a partir del fútbol como herramienta principal.

Valdría la pena no sólo fortalecer los estudios referentes al fútbol y el deporte en general, sino también hacer pleno uso de su profesionalización y sus beneficios como política pública para la atención de necesidades al corto, mediano y largo plazo, además de fomentar su apoyo y lograr interpretar todas estas interacciones las cuales carecen de observación y estudio.

Como afirmó el profesor Cesar Niño para nuestro proyecto Fútbol y Disciplina, "Sería grandioso, que así como existe en la Ciencia Política y las Relaciones Internacionales teorías feministas, naturalistas, de migraciones, existiera una dedicada al fútbol. Los aportes a la disciplina sé que serían continuos, tectónicos y numerosos." Pues a manera de Softpower el fútbol es un potencial elemento del cual se puede sacar mucho provecho, además de ser el eje de varios fenómenos sociales.

No en vano la prensa habla de los deportistas colombianos como embajadores en cada uno de sus países, en incluso un comentarista deportivo fue nombrado embajador en Sudáfrica a puertas de su primer y único mundial en la historia del continente africano, sin mencionar a Falcao García, que al igual que Drogba, fue mencionado como embajador de buena voluntad. 


\section{Bibliografía}

ALVAREZ SOBERANIS, J. (s.f.). El Problema de la Definición de la Seguridad Socialy el Derecho. Mèxico D.F: Escuela de Derecho de la Universidad Iberoamericana.

BALDWIN, D. (1980). Interdependence and power: a conceptual analysis. Wisconsin: Board of Regents of the University of Wisconsin System.

BLOYCE, D., y Smith, A. (2012). Polita Pública y Desarrollo del Deporte. (C. Parra Dussan, A. Quiñones Valero, Edits., A. Quiñones Valero, C. Parra Dussan, y J. Sierra Reyes, Trads.) New York, New York, Estados Unidos: Comité Paralímpico Colombiano.

BONIFACE:(31 de Mayo de 2006). Project-Syndicate. Recuperado el 20 de Juno de 2012, de La geopolítica del Fútbol: http://www.project-syndicate.org/commentary/the-geopoliticsof-football/spanish

BRICEÑO, G. (27 de Septiembre de 2008). El Tiempo. Recuperado el 10 de Julio de 2014, de http://www.eltiempo.com/archivo/documento/CMS-4570650

CAYUELA MALDONADO, J. (1997). Olimpyc Studies. Recuperado el 25 de Julio de 2014, de http://olympicstudies.uab.es/pdf/wp060_spa.pdf

DUQUE SOTO, L. (2 de Septiembre de 2011). El Tiempo. (El Tiempo) Recuperado el 2 de Agosto de 2014, de http:/www.eltiempo.com/archivo/documento-2013/CMS-10283204

DURÁN GONZÁLEZ, J. (2012). El vandalismo en el fútbol. Una reflexión sobre la violencia en la sociedad moderna. Madrid, España: Gymnos.

FAO. (2011). Food Security. (P. CE-FAO, Ed.) Recuperado el 16 de Julio de 2014, de http://www.foodsec.org/

FUNDACIÓN DEPORTIVA JACKSON MARTINEZ (2014). Fudejam. Recuperado el 3 de Agosto de 2014, de http://www.fudejam.org/

FÚTBOL PARA EL FUTURO. (2012). Fútbol para el futuro. Recuperado el 3 de Agosto de 2014, de http://www.futbolparaelfuturo.org/\#!modelo/c4f1

GUZMÁN, J. (Junio de 2002). Envejecimiento y desarrollo en América Latina y el Caribe. 
(C.-D. d. CEPAL, Ed.) Serie Población y Desarrollo en América Latina y el Caribe(28).

GOMIS, Josep (1989). "La violencia en el deporte "en Análisis Jurídico de la violencia en el deporte. Col-legi d'advocats de Barcelona. Barcelona.

HUENCHUAN, S., y Guzmán, J. M. (14 y 15 de Noviembre de 2006). CEPAL. (C.-D. d. Población, Ed.) Recuperado el 14 de Julio de 2014

HUERTA ROJAS, F. (Junio de 2002). Redalyc. Recuperado el 20 de Julio de 2014, de http://www.redalyc.org/pdf/325/32511306.pdf

MINUTO 116. (11 de Marzo de 2014). Didier Drogba, el 'elefante' que paró una guerra. Recuperado el 12 de Mayo de 2014, de Didier Drogba, el 'elefante' que paró una guerra: http:// revistaminuto116.com/didier-drogba-guerra-costa-de-marfil/

MEDINA, Xavier (1994). "El deporte como factor en la construcción sociocultural de la identidad", en VV.AA, Ciencias Sociales y Deporte, Investigación social y deporte, AEISAD $\mathrm{n}^{\mathrm{o}} 1$, Pamplona.

ORGANIZACIÓN INTERNACIONAL DEL TRABAJO (2001). Organización Internacional del trabajo. Recuperado el 14 de Julio de 2014

PARLAMENTO EUROPEO (1987). Dictamen sobre vandalismo y violencia en el deporte. Comisión de Juventud, Cultura, Educación, Información y Deporte. Noviembre

RECASÉNS, A. Las Barras Bravas. Facultad de Ciencias Sociales Universidad de Chile.

SUÁREZ REYES, F. (2010). Buenaventura: una ciudad-puerto, globalizante, diversa y multicultural. XIV Encuentro de Latinoamericanistas Españoles, (págs. 2488-2507). Universidad del Pacífico de Colombia.

UNISPORT (1992). I Encuentro sobre sociología del deporte", en Apuntes UNISPORT Andalucia, n²56. UNISPORT. Málaga. 
